\title{
Protocol for a systematic review and meta-analysis of cognitive-behavioural therapy for social anxiety disorder in psychosis
}

\author{
Maria Michail ${ }^{1 *}$, Max Birchwood $^{2}$ and Lynda Tait ${ }^{3}$
}

\begin{abstract}
Background: Social anxiety is among the most prevalent and debilitating affective disturbances manifest in people with psychosis. It is usually accompanied by high levels of depression and leads to significant social disability, lower quality of life and poorer prognosis as it raises the possibility of an early relapse. Despite its elevated prevalence and severity in psychosis, social anxiety remains under-recognized and under-treated. Cognitive-behavioural therapy is recommended for the treatment of people with psychosis. However, its focus and evaluation has primarily revolved around the reduction of psychotic symptoms, and not for co-morbid affective disturbances such as social anxiety. There is lack of evidence on the clinical effectiveness and cost-effectiveness of cognitive-behavioural interventions for the treatment of social anxiety disorder in psychosis.

Methods/Design: Electronic databases will be systematically searched for randomised controlled trials and quasi-experimental studies investigating the effectiveness and cost-effectiveness of cognitive-behavioural interventions for the treatment of social anxiety disorder in people with psychosis. Grey literature will also be searched by screening trial registers. Only studies published in English will be included in the review. Date restrictions will not be applied. Eligible studies will have as the primary outcome social anxiety (continuous data) measured using any psychometrically validated scale both self-reported and clinician administered. Secondary outcomes will include general anxiety symptoms, distress, depression, positive and negative symptoms of schizophrenia, and quality of life measured using any psychometrically validated scale, both self-reported and clinician administered, and the cost of cognitive-behaviour therapy (CBT) intervention (with another treatment or treatment-as-usual).

Conclusions: This review will provide an evidence synthesis of the effectiveness and cost-effectiveness of cognitive-behavioural interventions for the treatment of social anxiety disorder in people with psychosis. The review will identify the specific intervention components associated with effectiveness which will facilitate the translation of the existing evidence to the development of new, targeted interventions optimising these components. In doing so, this review will provide recommendations for the treatment of social anxiety and associated distress in psychosis and will further inform the development of future interventions in this area.
\end{abstract}

Trial registration: PROSPERO registration number CRD42014009052.

Keywords: Systematic review, Meta-analysis, Social anxiety, Psychosis, Schizophrenia, Cognitive-behavioural therapy

\footnotetext{
*Correspondence: maria.michail@nottingham.ac.uk

${ }^{1}$ School of Health Sciences, Jubilee Campus, University of Nottingham,

Nottingham NG7 2TU, UK

Full list of author information is available at the end of the article
}

() Biomed Central (c) 2014 Michail et al.; licensee BioMed Central Ltd. This is an Open Access article distributed under the terms of the Creative Commons Attribution License (http://creativecommons.org/licenses/by/4.0), which permits unrestricted use, distribution, and reproduction in any medium, provided the original work is properly credited. The Creative Commons Public Domain Dedication waiver (http://creativecommons.org/publicdomain/zero/1.0/) applies to the data made available in this article, unless otherwise stated. 


\section{Background}

Social anxiety disorder is among the most frequently reported psychiatric disorders with a lifetime prevalence of $12 \%$ and a 12-month prevalence of 7.1\% [1]. According to the Diagnostic and Statistical Manual of Mental Disorders, Fifth Edition (DSM-5) [2], social anxiety disorder (previously known as social phobia) is defined as "a marked fear or anxiety about one or more social situations in which the individual is exposed to possible scrutiny by others. Examples include social interactions (e.g., having a conversation), being observed (e.g., eating or drinking), or performing in front of others (e.g., giving a speech)."

People with social anxiety desire to make a favourable impression during social encounters but at the same time doubt their ability to do so; they fear that they will be scrutinized and negatively evaluated due to perceived failed social performance. These fears lead people with social anxiety to avoid all or some social situations and in extreme cases this could lead to complete social isolation [3]. Exposure to the feared situation is almost always accompanied by physical symptoms, for example, sweating, trembling, heart racing, which could develop (although not necessarily) into panic attacks.

Social anxiety develops at an early age, usually during childhood or adolescence and, once established, follows a stable, chronic course if treatment is not initiated ([4,5]; Findings regarding the sociodemographic characteristics of social anxiety disorder support that this is more prominent among females [6-10] although there have been studies [11] which have failed to confirm such gender differences. Higher incident rates have been consistently observed among unmarried individuals usually coming from a lower socioeconomic background, with poorer educational attainment and higher unemployment rates $[6,7,10]$. The average duration of illness is approximately 29 years $[4,12]$ and the likelihood of a full remission or recovery is significantly lower compared to that of other anxiety disorders [12].

\section{Social anxiety disorder in psychosis}

Social anxiety is among the most prevalent and debilitating affective disturbances manifested in people with psychosis with rates ranging between $8 \%$ and $36 \%$ ([13-15]; [16,17]; [18,19]). Social anxiety is usually accompanied by high levels of depression ([19];) and leads to significant social disability [17], a reduced quality of life [16] and poorer prognosis as it raises the possibility of an early relapse [20]. Despite its elevated prevalence and severity in psychosis, social anxiety remains underrecognized and under-treated. One of the reasons for this could be that the exact relationship between social anxiety and psychotic symptoms is yet to be determined and available empirical findings remain inconclusive [21].
Although theoretical models and empirical evidence consistently point towards a link between general anxiety and positive symptoms of psychosis, predominantly paranoia and persecutory delusions [22], social anxiety appears to have a distinct quality and its relationship to paranoia and persecutory thinking is not straightforward. Four pathways have been proposed for the understanding of the ontogeny of social anxiety in psychosis $[19,23]$ :

1. Social anxiety predates the onset of paranoia and helps maintain persecutory beliefs

2. Social anxiety and paranoia develop concurrently in the early phase of psychosis and follow a similar course

3. Social anxiety develops as a consequence of paranoid beliefs

4. Social anxiety emerges as a response to the shame and social stigma attached to a diagnosis of mental illness

\section{Clinical implications}

Cognitive-behavioural therapy (CBT) is recommended by the National Institute for Health and Care Excellence [24] for people with psychosis. However, the focus and evaluation of CBT for psychosis has primarily revolved around the reduction of psychotic symptoms, and not for co-morbid depression and social anxiety $[23,25]$. There is lack of evidence on the clinical effectiveness and cost effectiveness of CBT interventions for the treatment of affective dysregulation and associated distress in psychosis and particularly so for social anxiety disorder.

\section{Aim}

This systematic review and meta-analysis will provide an evidence synthesis of the effectiveness and costeffectiveness of CBT interventions for the treatment of social anxiety disorder in people with psychosis. In doing so, this review will provide recommendations for the treatment of social anxiety and associated distress in psychosis and will further inform the development of future interventions in this area. To the best of the authors' knowledge this is the first systematic review of CBT interventions for social anxiety in psychosis.

\section{Objectives}

Our objectives are:

1. To synthesize the evidence of the effectiveness and cost-effectiveness of CBT interventions for the treatment of social anxiety disorder in people with psychosis in improving outcomes related to social anxiety symptoms, general anxiety, distress, depression, positive and negative symptoms of schizophrenia, and quality of life. 
2. To estimate the aggregate effectiveness of CBT interventions for the treatment of social anxiety disorder in people with psychosis in improving outcomes related to social anxiety symptoms, general anxiety, distress, depression, positive and negative symptoms of schizophrenia, and quality of life.

\section{Methods/Design}

\section{Study design}

We shall conduct a systematic review and meta-analysis and we will report the outcomes according to the Preferred Reporting Items for Systematic Reviews and Meta-Analyses (PRISMA) statement [26] following the guidelines in the Cochrane Handbook for Systematic Reviews of Interventions [27].

\section{Search strategy}

The literature search strategy is included in Additional file 1. The following search limits will be set: 1) Study design: randomised controlled trials (RCTs) and quasiexperimental studies, 2) limited to English language. Date restrictions will not be applied. The following bibliographic databases will be searched:

- Cochrane Central Register of Controlled Trials

- CINAHL (Cumulative Index to Nursing and Allied

- Health Literature)

- EMBASE

- MEDLINE

- PsychINFO

- SCI (Science Citation Index)

\section{Grey literature}

- Clinical Trials: clinicaltrials.gov

- ISRCTN Register

\section{Search terms}

We shall use Medical Subject Headings (MeSH) and free-text word terms, as appropriate to the databases. The electronic search strategy terms (where necessary using wildcards) are:

\author{
Social anxiety \\ Social phobia \\ Social anxiety disorder \\ Schizophrenia \\ Psychosis \\ Cognitive therapy \\ CBT \\ Effectiveness \\ Treatment efficacy
}

Search strategies will be pilot tested. We shall use EndNote to record titles and abstracts for retrieval and inclusion/exclusion decisions.

\section{Selection criteria Types of participants}

Participants will be between 16 and 65 years of age, with schizophrenia or related psychoses (as diagnosed using any recognized diagnostic criteria) with social anxiety disorder as diagnosed using any recognised diagnostic criteria, for example, ICD-10 [28] or DSM-5 [2]. Studies including participants $<16$ yrs or $>65$ yrs or participants with a primary diagnosis of organic brain disorder will be excluded from this review.

\section{Types of studies}

RCTs and quasi-experimental studies will be included in the review.

\section{Types of intervention}

The review will include cognitive-behavioural (or cognitive) interventions that are targeted at social anxiety in people with psychosis. There will be no limitation in terms of psychological theory informing the intervention, the person delivering the intervention or the setting in which the intervention is delivered. Group and one-toone interventions will be included.

\section{Comparator}

Control conditions will include any other treatment, no treatment, treatment-as-usual and a waiting list control.

\section{Types of outcomes \\ Primary outcome}

Eligible studies will have as primary outcome social anxiety (continuous data) measured using any psychometrically validated scale, both self-reported and clinician administered.

\section{Secondary outcomes}

These will include general anxiety symptoms, distress, depression, positive and negative symptoms of schizophrenia and quality of life measured using any psychometrically validated scale both self-reported and clinician administered, and the cost of CBT intervention (with another treatment or treatment-as-usual).

\section{Selection procedure}

Two researchers (MM and LT) will independently screen the title and abstract of retrieved references for inclusion. The full text of all potential eligible studies will be obtained by MM. The next step will involve two researchers (MM and LT) independently assessing obtained references for inclusion. We shall pilot test the 
procedure on a small number of studies. Any disagreements will be resolved by consensus.

\section{Managing references}

Bibliographic software (EndNote) (Thomson Reuters), will be used to manage retrieved references. MM will be responsible for identifying and removing duplicates, ordering and recording the receipt of any inter-library loans and obtaining the full-text papers. MM will be the sole team member responsible for adding or amending library records in EndNote.

\section{Data extraction, procedures and data management}

The EPOC data extraction form in combination with the EPOC data checklist (see Additional file 2) will be used to extract data from relevant studies. Two reviewers (MM and LT) will work independently to extract data. Disagreements between the two reviewers will be resolved by discussion and consensus, or resolved by a third author (MB). Data extraction will include study setting, study population and participant demographics and baseline characteristics, details of the intervention and control conditions, study methodology, recruitment and study completion rates, outcomes and times of measurement, indicators of acceptability to users, suggested mechanisms of intervention action, information for assessment of the risk of bias and variables related to study quality. Study authors will be contacted to request data missing on methods or results. Information on missing data and dropouts will be assessed for each study. We will report the number of participants included in the final analysis of each study as a proportion of all participants in the study. The possible effects of the missing data will be discussed. Data will be included only for those participants whose results are known.

\section{Dealing with missing data}

Study authors will be contacted to request data missing on methods or results. Information on missing data and dropouts will be assessed for each study. We will report the number of participants included in the final analysis of each study as a proportion of all participants in the study. The possible effects of the missing data will be discussed. Data will be included only for those participants whose results are known.

\section{Quality assessment}

We shall assess the quality of studies and assessment of bias using the Cochrane's Collaboration tool for assessing risk of bias presented in Additional file 3 [29]. Two researchers (MM and LT) will independently rate risk of bias of each study. Discrepancies will be resolved by discussion and consensus.

\section{Data analysis}

\section{Measures of treatment effects}

We shall summarise and describe the characteristics of the population, interventions and outcomes, using descriptive statistics. Standardised mean differences, Hedges $g$, and weighting studies using inverse of variance will be calculated for continuous outcomes. Risk ratios (RR) will be calculated and we shall use the MantelHaenszel method to combine studies. We shall report outcomes using 95\% confidence intervals (CI), with random-effects models.

\section{Assessment of reporting biases}

Funnel plots will be drawn to investigate the relationship between study power and effect size. Possible reasons for any asymmetry will be discussed.

\section{Data synthesis and assessment of heterogeneity}

A meta-analysis of RCTs and quasi-experimental studies will be conducted using RevMan.

Statistical tests of heterogeneity (chi-square and Isquare) will be carried out. A random-effects model will be used to allow for expected heterogeneity. Effect estimates will be weighted by the inverse of their variance, giving greater weight to larger trials.

Sub group analyses will be carried out for studies with similar research questions based on: a) type of interventions: group-based CBT; individual CBT and b) phase of illness: early onset psychosis vs. chronic.

Sensitivity analysis will be carried out to explore the effects of the addition or removal of lower quality studies.

We will use the GRADE system 11 [30] to assess confidence in the quality of evidence of individual outcomes and strength of recommendations.

\section{Discussion}

CBT is recommended by the National Institute for Health and Care Excellence [24] for people with psychosis. However, its focus and evaluation for psychosis has primarily revolved around the reduction of psychotic symptoms, and not for co-morbid depression and social anxiety $[23,25]$. There is lack of evidence on the clinical effectiveness and cost effectiveness of CBT interventions for the treatment of affective dysregulation and associated distress in psychosis and particularly so for social anxiety disorder. This review will therefore provide an evidence synthesis of the effectiveness and costeffectiveness of CBT interventions for the treatment of social anxiety disorder in people with psychosis. The review will identify the specific intervention components associated with effectiveness which will facilitate the translation of the existing evidence to the development of new, targeted interventions optimising these components. 
In doing so, this review will provide recommendations for the treatment of social anxiety and associated distress in psychosis and will further inform the development of future interventions in this area. To the best of the authors' knowledge, this is the first systematic review of CBT interventions for social anxiety in psychosis.

\section{Additional files}

Additional file 1: Literature search strategy template. The file provides a description of the literature search strategy.

Additional file 2: Data abstraction form. Form that will be used by the authors to extract data.

Additional file 3: Quality assessment tool. Tool that will be used by the authors to assess risk of bias.

\section{Competing interests}

The authors declare that they have no competing interests.

\section{Authors' contributions}

MM conceived and designed the protocol. LT contributed elements to the design of the protocol. MM and LT jointly wrote the manuscript. All authors (MM, MB and $L T$ ) read and approved the final manuscript.

\section{Acknowledgements}

Professor Birchwood is partly funded by the National Institute for Health Research (NIHR) through the Collaborations for Leadership in Applied Health Research and Care West Midlands initiative. The views expressed are those of the authors and not necessarily those of the CLAHRC WM collaborative organisations, the NIHR or the Department of Health.

\section{Author details}

${ }^{1}$ School of Health Sciences, Jubilee Campus, University of Nottingham, Nottingham NG7 2TU, UK. 'Warwick Medical School, University of Warwick, Coventry CV4 7AL, UK. ${ }^{3}$ School of Health Sciences, University of Nottingham, Nottingham NG7 2HA, UK.

Received: 26 March 2014 Accepted: 19 May 2014

Published: 11 June 2014

\section{References}

1. Kessler RC, Berglund P, Demler O, Jin R, Merikangas KR, Waters EE: Lifetime prevalence and age-of-onset distributions of DSM-IV disorders in the National Comorbidity Survey Replication. Arch Gen Psychiatry 2005, 62:593-602. Erratum in: Arch Gen Psychiatry 2005, 62:768. Merikangas, Kathleen R [added].

2. American Psychiatric Association: Diagnostic and Statistical Manual of Mental Disorders. 5th edition. Arlington, VA: American Psychiatric Publishing; 2013.

3. Clark DM, Wells A: A cognitive model of social phobia. In Social Phobia. Edited by Heimberg L. New York: Guildford Press; 1995.

4. Chartier MJ, Hazen AL, Stein MB: Lifetime patterns of social phobia: a retrospective study of the course of social phobia in a nonclinical population. Depress Anxiety 1998, 7:113-121.

5. Yonkers KA, Dyck IR, Keller MB: An eight-year longitudinal comparison of clinical course and characteristics of social phobia among men and women. Psychiatr Serv 2001, 52:637-643.

6. Schneier FR, Johnson J, Hornig CD, Liebowitz MR, Weissman MM: Social phobia. Comorbidity and morbidity in an epidemiological sample. Arch Gen Psychiatry 2002, 49:282-288.

7. Davidson JR, Hughes DL, George LK, Blazer DG: The epidemiology of social phobia: findings from the Duke Epidemiological Catchment Area Study. Psychol Med 1999, 23:709-718.

8. Kessler RC, McGonagle KA, Zhao S, Nelson CB, Hughes M, Eshleman S, Wittchen $\mathrm{H}-\mathrm{U}$, Kendler KS: Lifetime and 12-month prevalence of DSM-III-R psychiatric disorders in the United States. Results from the National Comorbidity Survey. Arch Gen Psychiatry 1994, 51:8-19.
9. Wittchen $\mathrm{H}-\mathrm{U}$, Stein MB, Kessler RC: Social fears and social phobia in a community sample of adolescents and young adults: prevalence, risk factors and co-morbidity. Psychol Med 1999, 29:309-323.

10. Magee WJ, Eaton WW, Wittchen HU, McGonagle KA, Kessler RC: Agoraphobia, simple phobia, and social phobia in the National Comorbidity Survey. Arch Gen Psychiatry 1996, 53:159-168.

11. Stein M, Torgrud $J$, Walker RJ: Social phobia subtypes, symptoms and severity. Arch Gen Psychiatry 2000, 57:1046-1105.

12. Keller MB: The lifelong course of social anxiety disorder: a clinical perspective. Acta Psychiatr Scand Suppl 2003, 108:85-94.

13. Cosoff SJ, Hafner RJ: The prevalence of comorbid anxiety in schizophrenia, schizoaffective disorder and bipolar disorder. Aust N Z J Psychiatry 1998, 32:67-72.

14. Cassano GB, Pini S, Saettoni M, Dell'Osso L: Multiple anxiety disorder comorbidity in patients with mood spectrum disorders with psychotic features. Am J Psychiatry 1999, 156:474-476.

15. Goodwin RD, Amador XF, Malaspina D, Yale SA, Goetz RR, Gorman JM: Anxiety and substance use comorbidity among inpatients with schizophrenia. Schizophr Res 2003, 61:89-95.

16. Pallanti S, Quercioli L, Hollander E: Social anxiety in outpatients with schizophrenia: a relevant cause of disability. Am J Psychiatry 2004 161:53-58.

17. Voges M, Addington J: Association between social anxiety and social functioning in first episode psychosis. Schizophr Res 2005, 76:287-292.

18. Mazeh D, Bodner E, Weizman R, Delayahu Y, Cholostoy A, Martin T, Barak Y: Co-morbid social phobia in schizophrenia. Int J Soc Psychiatry 2009, 55:198-202.

19. Michail M, Birchwood M: Social anxiety disorder in first-episode psychosis: incidence, phenomenology and relationship with paranoia. Br J Psychiatry 2009, 195:234-241

20. Gumley A: Staying well after psychosis: a cognitive interpersonal approach to emotional recovery after relapse prevention. Tidsskrift Norsk Psykologforening 2007, 44:667-676.

21. Michail M: Social anxiety disorder in psychosis: a critical review. In New Insights into Anxiety Disorders. Edited by Durbano F. Rijeka, Croatia: InTech- Open Access Publisher; 2013.

22. Freeman D, Garety PA, Kuipers E: Persecutory delusions: developing the understanding of belief maintenance and emotional distress. Psychol Med 2001, 31:1293-1306.

23. Michail M, Birchwood M: Social anxiety and shame cognitions in psychosis Psychol Med 2013, 43:133-142.

24. National Institute for Health and Care Excellence: Psychosis and schizophrenia: treatment and management. (Clinical guideline 178.). 2014. http://guidance.nice. org.uk/CG178].

25. Michail M, Birchwood M: Social anxiety in first-episode psychosis: the role of childhood trauma and adult attachment. J Affect Disord 2014, 163:102-109.

26. Moher D, Liberati A, Tetzlaff J, Altman DG, The PRISMA Group (2009): Preferred Reporting Items for Systematic Reviews and Meta-Analyses: The PRISMA Statement. BMJ 2009, 339:b2535.

27. Higgins JP, Green S: Cochrane handbook for systematic reviews of interventions, Volume 5. Chichester, UK: John Wiley and Sons (Wiley Online Library); 2008.

28. World Health Organisation: The ICD-10 Classification of Mental and Behavioural Disorders. Geneva: World Health Organisation; 1993.

29. Higgins JP, Altman DG, Gøtzsche PC, Jüni $P$, Moher D, Oxman AD, Savovic J, Schulz KF, Weeks L, Sterne JA: The Cochrane's collaboration tool for assessing risk of bias in randomised trials. BMJ 2011, 343:d5928.

30. Atkins D, Best D, Briss PA, Eccles M, Falck-Ytter Y, Flottorp S, Guyatt GH, Harbour RT, Haugh MC, Henry D, Hill S, Jaeschke R, Leng G, Liberati A, Magrini N, Mason J, Middleton P, Mrukowicz J, O'Connell D, Oxman AD, Phillips B, Schünemann HJ, Edejer T, Varonen $H$, Vist GE, Williams JW Jr, Zaza S, GRADE Working Group: Grading quality of evidence and strength of recommendations. BMJ 2004, 328:1490.

doi:10.1186/2046-4053-3-62

Cite this article as: Michail et al:: Protocol for a systematic review and meta-analysis of cognitive-behavioural therapy for social anxiety disorder in psychosis. Systematic Reviews 2014 3:62. 